\title{
A Reduced Model for Flame-Flow Interaction
}

\author{
P. Gordon ${ }^{a}$, M. Frankel ${ }^{b}$ and G. Sivashinsky ${ }^{c 1}$ \\ ${ }^{a}$ Department of Mathematical Sciences, New Jersey Institute of Technology, \\ University Heights, Newark, NJ 07102, USA \\ ${ }^{b}$ Department of Mathematical Sciences, Indiana University Purdue University, \\ Indianapolis, IN 46202, USA \\ ${ }^{c}$ Department of Mathematical Sciences, Tel Aviv University, Tel Aviv 69978, Israel
}

\begin{abstract}
The paper is concerned with an extension of the classical relation between the flame speed and the curvature-flow stretch, valid only for high Lewis numbers (diffusively stable flames). At low Lewis numbers the corresponding flame-flow system suffers shortwavelength instability, making the associated initial value problem ill-posed. In this study the difficulty is resolved by incorporation of higher-order effects. As a result one ends up with a reduced model based on a coupled system of second-order dynamic equations for the flame interface and its temperature. As an illustration the new model is applied for description of diffusively unstable stagnation-point flow flames.
\end{abstract}

Key words: premixed flames, instabilities in flames, flame stretch

AMS subject classification: 80A25, 35K57, 35B40

\section{Introduction}

In premixed gas combustion it is often reasonable to view a propagating flame as an interface convected by the underlying flow field while advancing towards the unburned gas at a normal velocity $v_{F}$. In the constant density limit when the flow-field $\mathbf{u}(\mathbf{x}, t)$ is prescribed (passive propagation) the flame dynamics is governed by the equation,

$$
v_{n}-\mathbf{u} \cdot \mathbf{N}=v_{F}, \quad \nabla \cdot \mathbf{u}=0
$$

\footnotetext{
${ }^{1}$ Corresponding author. Email: grishas@post.tau.ac.il
} 
Here $v_{n}$ is the normal velocity of the interface relative to the laboratory frame of reference, $\mathbf{N}$ is the unit normal to the interface directed towards the unburned gas, and $v_{F}$ is the normal velocity of the interface relative to a local fluid element; $v_{F}$ depends on the local curvature of the interface as well as the local strain field.

For weakly curved flames evolving through large-scale flow fields [1], [2],

$$
v_{F}=v_{L}\left(1-l_{M} \mathcal{K}\right)
$$

where $v_{L}$ represents the laminar flame velocity in the absence of strain and curvature effects. $\mathcal{K}$ is the stretch, the combined effect of curvature and strain. The relation specifying the stretch may be written as [2],

$$
v_{L} \mathcal{K}=-v_{L} \varkappa-\mathbf{N} \cdot \mathbf{E} \cdot \mathbf{N}
$$

Here $\mathbf{E}=\frac{1}{2}\left(\nabla \mathbf{u}+\nabla \mathbf{u}^{T}\right)$ is the rate of strain tensor evaluated at the interface, $\varkappa=-\nabla \cdot \mathbf{N}$ is the mean curvature of the interface and $l_{M}$ is the Markstein length whose value depends on physico-chemical parameters of the system. In the constant density, high activation energy, near-equidiffusive approximation [3],

$$
l_{M}=l_{t h}\left(1+\frac{1}{2} \beta(\mathrm{Le}-1)\right)
$$

where $l_{t h}$ is the planar flame thickness; $\beta$ and Le are the Zeldovich and Lewis numbers, respectively. Eqs. (1.1)-(1.3) define a second-order differential equation. For positive $l_{M}$ (high Lewis numbers) the short-wavelength disturbances decay exponentially ensuring the well-posedness of the associated initial-value problem. However, if $l_{M}$ is negative (small Lewis numbers) the short-wavelength disturbances undergo exponential growth, making the intialvalue problem ill-posed, and the whole model incapable of securing a sensible dynamical picture.

The short-wavelength instability is a trace of the well-known flame cellularity occurring at low Lewis numbers. An adequate description of cellular flames requires incorporation of higher-order effects absent in the first-order model based on Eqs. (1.1)-(1.3). Such higherorder models have long been developed for the flames evolving in quiescent mixtures. Here, depending on the asymptotic strategy adopted, one ends up either with a single fourthorder equation for the flame-interface [4], [5] or with a system of second-order equations for the flame interface and its temperature [6],[7]. The objective of the present work is an extension of the existing higher-order models to incorporate effects due to the background flow-fields, which, to our knowledge, has not previously been undertaken. The present paper is a development of our recent study on the subject [8], restricted to the 2D geometry, over the general 3D situation. Moreover, in [8] the problem was tackled heuristically as a geometrically invariant extrapolation of the results obtained for freely evolving flames. In the present paper the problem is solved in a more rational manner by means of an appropriate gradient expansion in the intrinsic coordinates.

The paper is organized as follows. In Section 2 we present basic equations for the flame dynamics in the constant density approximation.In Section 3 we derive a reduced model for 
the flame interface evolution and discuss basic properties of the system. Section 4 deals with the numerical simulation. In Appendix we present some relations from the differential geometry.

\section{Formulation}

As a starting point we adopt the conventional constant density, high activation energy, reaction-diffusion-advection formulation where the reaction rate is modeled by the surface $\delta$-function, $\delta_{\sigma}$. In suitably chosen units, the corresponding system of equations reads,

$$
\begin{aligned}
& T_{t}+\mathbf{U} \cdot \nabla T=\Delta T+\exp \left(\frac{1}{2} \beta(T-1)\right) \delta_{\sigma} \\
& C_{t}+\mathbf{U} \cdot \nabla C=\mathrm{Le}^{-1} \Delta C-\exp \left(\frac{1}{2} \beta(T-1)\right) \delta_{\sigma}
\end{aligned}
$$

Here $T$ and $C$ are the appropriately scaled temperature and the deficient reactant concentration. In the constant density approximation the flow field $\mathbf{U}$ is incompressible, that is,

$$
\nabla \cdot \mathbf{U}=0
$$

Far ahead of the interface $T=0$ and $C=1$. Far behind the temperature approaches the adiabatic temperature of combustion products $T=1 ; C=0$ on and behind the interface. That is,

$$
\begin{aligned}
& T(\boldsymbol{\xi}, n, t) \rightarrow 0, \quad C(\boldsymbol{\xi}, n, t) \rightarrow 1 \quad \text { as } \quad n \rightarrow \infty \\
& T(\boldsymbol{\xi}, n, t) \rightarrow 1 \quad \text { as } \quad n \rightarrow-\infty \\
& C(\boldsymbol{\xi}, n, t)=0 \quad \text { at } \quad n \leq 0
\end{aligned}
$$

Here $n$ stands for the distance from the interface in its normal direction and $\boldsymbol{\xi}=\left(\xi_{1}, \xi_{2}\right)$ are local coordinates on the interface.

For further discussion it is convenient to work with a transformed version of the system (2.1) based on the scaled enthalpy,

$$
H=T+C
$$

rather than on the concentration.

If initially $H=1$ and $\mathrm{Le}=1$, then $H=1$ for all times. In this paper we consider the near-equidiffusive limit when Le is close unity and $\beta$ is large, but $\alpha=\frac{1}{2} \beta(1-$ Le) is finite. In this case $T$ and $H$ can be sought as power series of $2 / \beta$ :

$$
\begin{aligned}
& T=T_{0}+\left(\frac{2}{\beta}\right) T_{1}+\cdots \\
& H=H_{0}+\left(\frac{2}{\beta}\right) H_{1}+\cdots
\end{aligned}
$$


Substituting the anzatz (2.5) into (2.1), for the leading order approximation the original system (2.1) reduces to [3],

$$
\begin{aligned}
& T_{t}+\mathbf{U} \cdot \nabla T=\Delta T+\exp (\Theta) \delta_{\sigma}, \\
& S_{t}+\mathbf{U} \cdot \nabla S=\Delta(S-\alpha T),
\end{aligned}
$$

Here $T=T_{0}$ and $S=H_{1}$. $\Theta$ is $S$ evaluated at the interface, and may be viewed as a scaled excess temperature of the interface.

The boundary conditions for the system (2.6) read,

$$
\begin{aligned}
& T(\boldsymbol{\xi}, n, t) \rightarrow 0, \quad S(\boldsymbol{\xi}, n, t) \rightarrow 0 \quad \text { as } \quad n \rightarrow \infty, \\
& T(\boldsymbol{\xi}, n, t)=1 \quad \text { and } \quad S(\boldsymbol{\xi}, n, t) \quad \text { grows no faster than polynomially for all } n \leq 0 .
\end{aligned}
$$

Note that the system (2.6) is identical to the following formulation,

$$
\begin{aligned}
& T_{t}+\mathbf{U} \cdot \nabla T=\Delta T \\
& S_{t}+\mathbf{U} \cdot \nabla S=\Delta(S-\alpha T),
\end{aligned}
$$

involving jump and continuity conditions on the interface rather than the surface $\delta$-function.

$$
\begin{aligned}
& {\left[\partial_{n} S(\boldsymbol{\xi}, 0, t)\right]=\alpha\left[\partial_{n} T(\boldsymbol{\xi}, 0, t)\right]=-\alpha \exp (\Theta(\boldsymbol{\xi}, t)),} \\
& {[S(\boldsymbol{\xi}, 0, t)]=0, \quad T(\boldsymbol{\xi}, 0, t)=1, \quad \Theta(\boldsymbol{\xi}, t)=S(\boldsymbol{\xi}, 0, t) .}
\end{aligned}
$$

\section{Derivation of the reduced model}

In this section we derive an asymptotic model for the dynamics of the flame interface governed by the system (2.8), (2.9).

We start from geometrical settings. Let $\left(\xi_{1}, \xi_{2}, n\right)$ be an orthogonal system of curvilinear coordinates, and define the position vector (Fig.1),

$$
\mathbf{r}=\mathbf{R}\left(\xi_{1}, \xi_{2}, t\right)+n \mathbf{N}\left(\xi_{1}, \xi_{2}, t\right)
$$

Here $\xi_{i}$ are local coordinates on the interface aligned with the principle directions of curvature, and $n$ is the distance from the interface. $\mathbf{N}$ is the unit normal to the interface and $t$ is time. Thus the interface is described by $\mathbf{r}$ evaluated at $n=0$, that is by $\mathbf{R}$.

Next we introduce an orthonormal basis $\left(\mathbf{N}, \mathbf{e}_{1}, \mathbf{e}_{2}\right)$ where $\mathbf{e}_{i}$ are defined as,

$$
\mathbf{e}_{i}=\frac{1}{a_{i}} \frac{\partial \mathbf{R}}{\partial \xi_{i}}, \quad a_{i}=\left\|\frac{\partial \mathbf{R}}{\partial \xi_{i}}\right\|
$$

We assume that the interface is weakly distorted and thus the mean curvature $k$ is small 


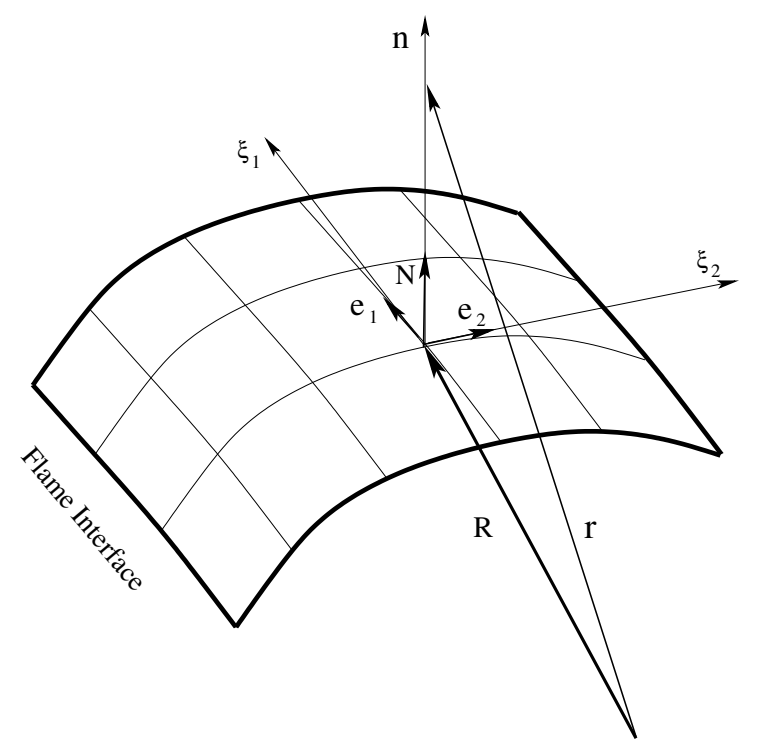

Figure 1: Parameterization of the flame interface

(say of the order of $\varepsilon$ where $\varepsilon<<1$ ), and the flow is slowly varying,

$$
\mathbf{U}=\mathbf{U}(\varepsilon t, \varepsilon n, \varepsilon \boldsymbol{\xi})
$$

This provides the natural scaling,

$$
k=\varepsilon \kappa, \quad \mathbf{s}=\varepsilon \boldsymbol{\xi}, \quad \tau=\varepsilon t
$$

which allows to expand the differential operators of the model $(2.8),(2.9)$ in power series of $\varepsilon$.

We also assume that the impact of terms involving powers of curvature and high order derivatives of the flow-field on the overall dynamics picture is not important and these terms may be ignored. This assumption is based on consideration of certain special flame-flow configurations near the stability threshold [4],[9], [10]. With these premises the differential operators assume the following form (see Appendix for details).

The convective time derivative, taking into account incompressibility condition, reads,

$$
\frac{\partial}{\partial t}+\mathbf{U} \cdot \nabla \approx\left(U_{n}-V_{n}\right) \frac{\partial}{\partial n}+\varepsilon n\left(\kappa \bar{U}_{n}-\bar{\nabla}_{\sigma} \cdot \overline{\mathbf{U}}_{\perp}\right) \frac{\partial}{\partial n}+\varepsilon \mathcal{D}_{\tau}
$$

where

$$
\begin{gathered}
\mathcal{D}_{\tau}=\frac{\partial}{\partial \tau}+\left(\overline{\mathbf{V}}_{\perp}+\overline{\mathbf{U}}_{\perp}\right) \cdot \bar{\nabla}_{\sigma}, \\
\bar{\nabla}_{\sigma}=\frac{\mathbf{e}_{1}}{a_{1}} \frac{\partial}{\partial s_{1}}+\frac{\mathbf{e}_{2}}{a_{2}} \frac{\partial}{\partial s_{2}}
\end{gathered}
$$

and

$$
\kappa=-\bar{\nabla}_{\sigma} \cdot \mathbf{N}
$$


The Laplace operator,

$$
\Delta \approx \frac{\partial^{2}}{\partial n^{2}}-\varepsilon \kappa \frac{\partial}{\partial n}+\varepsilon^{2} \bar{\Delta}_{\sigma}
$$

where

$$
\bar{\Delta}_{\sigma} \Phi=\bar{\nabla}_{\sigma} \cdot \bar{\nabla}_{\sigma} \Phi=\frac{1}{a_{1} a_{2}}\left(\frac{\partial}{\partial s_{1}}\left(\frac{a_{2}}{a_{1}} \frac{\partial}{\partial s_{1}} \Phi\right)+\frac{\partial}{\partial s_{2}}\left(\frac{a_{1}}{a_{2}} \frac{\partial}{\partial s_{2}} \Phi\right)\right) .
$$

Here $V_{n}=\mathbf{R}_{\tau} \cdot \mathbf{N}$ and $\overline{\mathbf{V}}_{\perp}=-\left(\mathbf{R}_{\tau} \cdot \mathbf{e}_{1}\right) \mathbf{e}_{1}-\left(\mathbf{R}_{\tau} \cdot \mathbf{e}_{2}\right) \mathbf{e}_{2}$ are normal and tangent components of the velocity of the interface. $\mathcal{L}_{\tau}=\partial_{\tau}+\overline{\mathbf{V}}_{\perp} \cdot \nabla_{\mathcal{S}}$ is the Lagrangian normal time derivative. $\bar{U}_{n}$, $\overline{\mathbf{U}}_{\perp}$ are normal and tangent component of the velocity of the flow velocity at the interface. That is $\overline{\mathbf{U}}(\tau, \mathbf{s})=\mathbf{U}(\tau, n=0, \mathbf{s})$ and $\bar{\nabla}_{\sigma}, \bar{\Delta}_{\sigma}$ are the surface gradient and Laplace-Beltrami operators.

It is important to note that the last term $\mathcal{D}_{\tau}$ in (3.5) is kept for regularization purposes. A similar role is played by the term $\bar{\Delta}_{\sigma}$ in the expansion of the Laplace operator $\Delta$, Eq.(3.9).

The problem $(2.8),(2.9)$ then can be solved by iterations. We expand the temperature, enthalpy and velocity in power series of $\varepsilon$ :

$$
\begin{aligned}
& T=T^{0}+\varepsilon T^{1}+\cdots, \quad S=S^{0}+\varepsilon S^{1}+\cdots, \\
& V_{n}=V_{n}^{0}+\varepsilon V_{n}^{1}+\cdots, \quad \Theta=\Theta^{0}+\varepsilon \Theta^{1}+\cdots
\end{aligned}
$$

The problem for the zeroth approximation (basic solution) then can be written as follows,

$$
\begin{aligned}
& \partial_{n}^{2} T^{0}+\left(V_{n}^{0}-\bar{U}_{n}\right) \partial_{n} T^{0}=0 \\
& \partial_{n}^{2} S^{0}+\left(V_{n}^{0}-\bar{U}_{n}\right) \partial_{n} S^{0}=\alpha \partial_{n}^{2} T^{0}
\end{aligned}
$$

with boundary, jump and continuity conditions,

$$
\begin{aligned}
& T^{0}(\mathbf{s}, n, \tau) \rightarrow 0, \quad S^{0}(\mathbf{s}, n, \tau) \rightarrow 0 \quad n \rightarrow \infty \\
& T^{0}(\mathbf{s}, n, \tau)=1, \quad S^{0}(\mathbf{s}, n, \tau) \quad \text { grows no faster than polynomially for all } n \leq(03.13) \\
& {\left[\partial_{n} S^{0}(\mathbf{s}, 0, \tau)\right]=\alpha\left[\partial_{n} T^{0}(\mathbf{s}, 0, \tau)\right]=-\alpha, \quad\left[S^{0}(\mathbf{s}, 0, \tau)\right]=0}
\end{aligned}
$$

The associated solution reads,

$$
\begin{aligned}
& T^{0}=e^{-n} \quad \text { for } \quad n>0 \quad V_{n}^{0}=1+\bar{U}_{n}, \\
& S^{0}=-\alpha n e^{-n} \quad \text { for } \quad n>0, \quad S^{0}=0, \quad T^{0}=1 \quad \text { for } \quad n \leq 0
\end{aligned}
$$

Higher order terms are obtained iteratively from the system of linear equations,

$$
\begin{aligned}
& \partial_{n}^{2} T^{m}+\partial_{n} T^{m}=F_{m}\left(T^{m-1}, \cdots, T^{0}, S^{m-1}, \cdots, S^{0}, n, \mathbf{s}, \tau\right) \\
& \partial_{n}^{2} S^{m}+\partial_{n} S^{m}=\alpha \partial_{n}^{2} T^{m}+G_{m}\left(T^{m-1}, \cdots, T^{0}, S^{m-1}, \cdots, S^{0}, n, \mathbf{s}, \tau\right)
\end{aligned}
$$

Here functions $F_{m}, G_{m}$ are obtained by substituting the anzatz (3.11) into the system (2.8) and equating terms with similar powers of $\varepsilon$. 
The equation for the evolution of the normal velocity $V_{n}$ is obtained using the basic solution (3.14). Indeed, since $T^{0}$ and $S^{0}$ are independent of $\mathbf{s}$ and $t$, one obtains

$$
\partial_{n}^{2} T+\left(V_{n}-\bar{U}_{n}\right) \partial_{n} T=\varepsilon\left(\kappa+\left(\kappa \bar{U}_{n}-\bar{\nabla}_{\sigma} \cdot \overline{\mathbf{U}}_{\perp}\right) n\right) \partial_{n} T^{0}+O\left(\varepsilon^{2}\right)
$$

Integration of (3.16) from 0 to $\infty$ yields,

$$
\begin{aligned}
& V_{n}=e^{\Theta}+\bar{U}_{n}-\varepsilon K+O\left(\varepsilon^{2}\right) \\
& K=-\left(1+\bar{U}_{n}\right) \kappa+\bar{\nabla}_{\sigma} \cdot \overline{\mathbf{U}}_{\perp}
\end{aligned}
$$

The derivation of the equation for $\Theta$ is more involved but still straightforward. It requires solving the system (3.15) for the first and second approximation. Upon matching corresponding solutions on the interface, using jump and continuity conditions (2.9), one obtains,

$$
\varepsilon \mathcal{D}_{\tau} \Theta=\varepsilon^{2} \bar{\Delta}_{\sigma} \Theta+\frac{\alpha}{4} K-\frac{1}{4} \Theta e^{\Theta}
$$

It is convenient to return to the original unscaled coordinates, which is formally equivalent to setting $\varepsilon=1$. The resulting system of governing equations then becomes,

$$
\begin{aligned}
& V_{n}=U_{n}+e^{\Theta}-K \\
& \mathcal{D}_{t} \Theta=\bar{\Delta}_{\xi} \Theta+\frac{\alpha}{4} K-\frac{1}{4} \Theta e^{\Theta}
\end{aligned}
$$

with

$$
\begin{aligned}
& K=-\left(1+\bar{U}_{n}\right) k+\bar{\nabla}_{\xi} \cdot \mathbf{U}_{\perp} \\
& \mathcal{D}_{t}=\partial_{t}+\left(\overline{\mathbf{U}}_{\perp}+\overline{\mathbf{V}}_{\perp}\right) \cdot \bar{\nabla}_{\xi}
\end{aligned}
$$

As shown in the Appendix the tangential (induced) velocity field $\overline{\mathbf{V}}_{\perp}$ is determined from the separate problem,

$$
\begin{aligned}
& \bar{\nabla}_{\xi} \cdot \overline{\mathbf{V}}_{\perp}=-k V_{n} \\
& \mathbf{e}_{1} \cdot \bar{\nabla}_{\xi} \overline{\mathbf{V}}_{\perp} \cdot \mathbf{e}_{2}+\mathbf{e}_{2} \cdot \bar{\nabla}_{\xi} \overline{\mathbf{V}}_{\perp} \cdot \mathbf{e}_{1}=0
\end{aligned}
$$

with $\mathbf{V}_{\perp}=0$ on the lateral boundary of the interface. Here $\mathbf{e}_{1}$ and $\mathbf{e}_{2}$ is a basis on the interface associated with the local coordinates $\left(s_{1}, s_{2}\right)$.

In the case of the two dimensional problem the system (3.19) becomes

$$
\begin{aligned}
& V_{n}=\bar{U}_{n}+e^{\Theta}-\tilde{K} \\
& \tilde{\mathcal{D}}_{t} \Theta=\partial_{s}^{2} \Theta+\frac{\alpha}{4} \tilde{K}-\frac{1}{4} \Theta e^{\Theta} \\
& \partial_{s} V_{s}=-\tilde{k} V_{n}
\end{aligned}
$$

with

$$
\begin{aligned}
& \tilde{K}=-\left(1+\bar{U}_{n}\right) \tilde{k}+\partial_{s} \bar{U}_{s} \\
& \tilde{\mathcal{D}}_{t}=\partial_{t}+\left(\bar{U}_{s}+V_{s}\right) \partial_{s}
\end{aligned}
$$

Here $\tilde{k}$ is the curvature and $s$ is the arc-length. $\bar{U}_{s}, V_{s}=d s / d t$ are tangential components of the external flow (computed at the interface) and the induced flow. 


\section{Cellular flames in the stagnation-point flow}

As an illustration, in this section, the model (3.22) is employed to describe the classical system of the diffusively unstable flame $(\alpha>1)$ held in the stagnation-point flow $\mathbf{U}(x, y)=$ $(q(x-L / 2),-q y)[9][11][12]$ (Fig. 2). Here $q$ is the appropriately scaled flow intensity. The

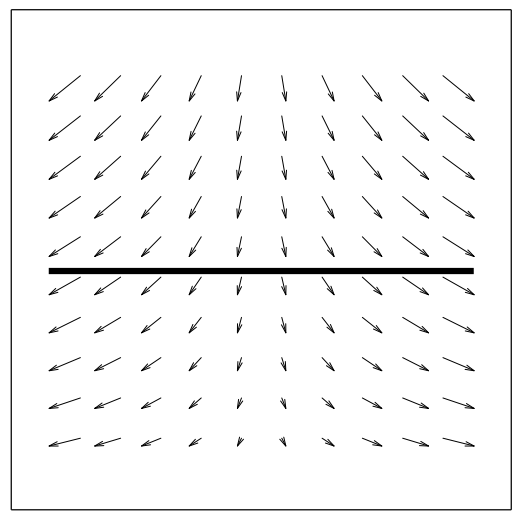

Figure 2: Diagram of premixed planar flame (bold line) stabilized in stagnation-point flow field. The upstream flow corresponds to the fresh mixture, the downstream to the burned gas.

system is considered over half-strip $(0<x<L, y<0)$ with the zero-gradient boundary conditions for the flame interface $y=F(x, \tau)$ and its excess temperature $\Theta(x, \tau)$,

$$
F_{x}(0, \tau)=\Theta_{x}(0, \tau)=0, \quad F_{x}(L, \tau)=\Theta_{x}(L, \tau)=0 .
$$

The problem is solved numerically for $\alpha=2.5, L=400,0.01 \leq q \leq 0.02$. It is found, as

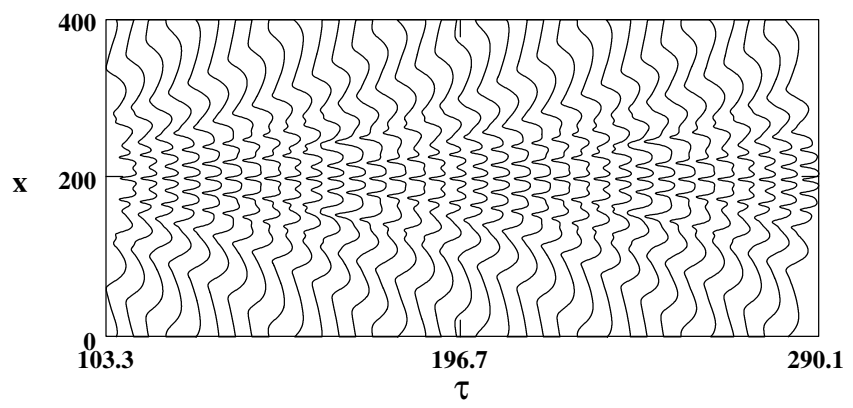

Figure 3: Temporal evolution of the cellular flame.

expected [12], that for sufficiently high flow intensity $(q=0.02)$ the flame interface is planar and located near $y=-1 / q$. However, if $q$ is small enough $(q=0.01)$ the central part of the flame becomes cellular with the cells in a state of permanent self-motion similar to that occurring in freely propagating cellular flames. 
Figure 3 shows the temporal evolution of the cellular flame at $q=0.014$, Figure 4 an instantaneous profiles of the flame interface and Figure 5 its temperature.

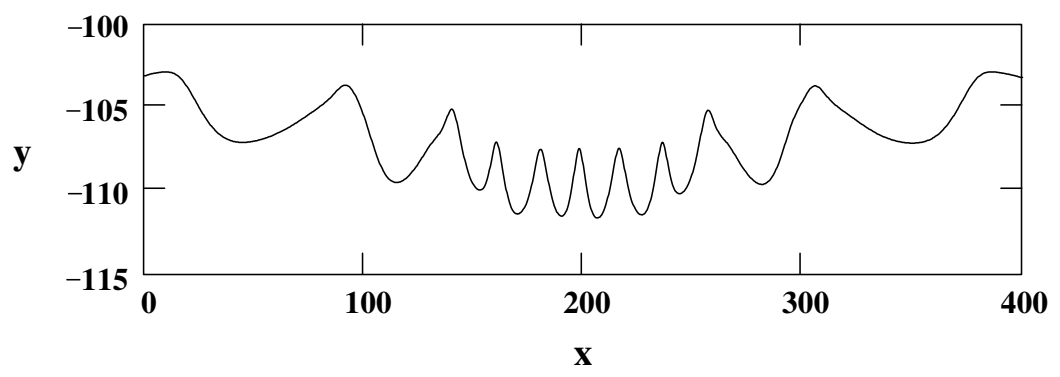

Figure 4: An instantaneous profile of the flame interface.

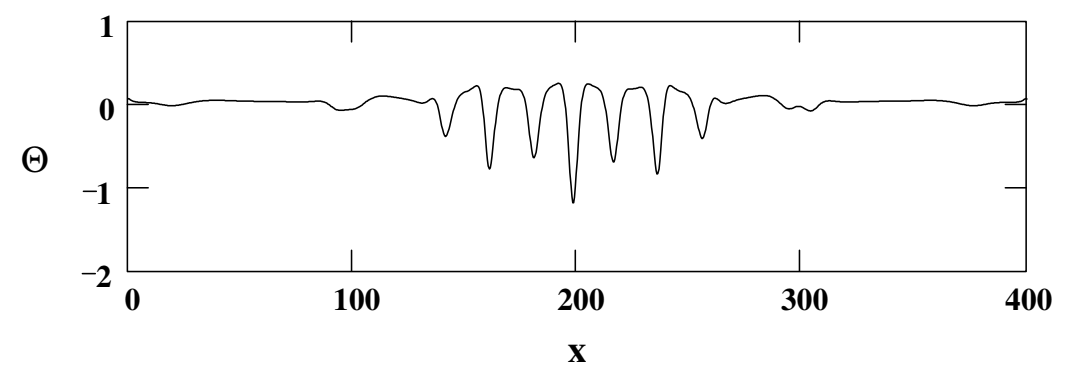

Figure 5: Temperature distribution for the profile in Fig.4

Acknowledgments. The work of PG was supported in part by the NSF Grant DMS0554775, MLF was partially supported by the NSF Grant DMS-0207308, and GIS was supported by the US-Israel Binational Science Foundation under Grant 2006-151, the Israel Science Foundation under Grant 350/05, and the European Community Program RTNHPRN-CT-2002-00274.

\section{Appendix A. Expressions for differential operators}

In this section we recall some results from the differential geometry Derivation of the basic formulas presented here can be found in [13], [14] and [15].

Let $\left(\xi_{1}, \xi_{2}, n\right)$ be an orthogonal system of curvilinear coordinates, and define the position vector,

$$
\mathbf{r}=\mathbf{R}\left(\xi_{1}, \xi_{2}, t\right)+n \mathbf{N}\left(\xi_{1}, \xi_{2}, t\right)
$$

In this setting $\xi_{i}$ are local coordinates on the interface and $n$ is the distance from the interface. $\mathbf{N}$ is the unit normal to the interface and $t$ is time. Thus, the interface is described by $\mathbf{r}$ 
evaluated at $n=0$, that is by $\mathbf{R}$. We introduce an orthogonal basis $\left(\mathbf{N}, \mathbf{e}_{1}, \mathbf{e}_{2}\right)$ where $\mathbf{e}_{i}$ are defined as,

$$
\mathbf{e}_{i}=\frac{1}{a_{i}} \frac{\partial \mathbf{R}}{\partial \xi_{i}}, \quad a_{i}=\left\|\frac{\partial \mathbf{R}}{\partial \xi_{i}}\right\|
$$

Surface gradient and Laplace-Beltrami operator

Define the gradient

$$
\nabla=\mathbf{N} \frac{\partial}{\partial n}+\nabla_{\xi}, \quad \nabla_{\xi}=\frac{\mathbf{e}_{1}}{l_{1}} \frac{\partial}{\partial \xi_{1}}+\frac{\mathbf{e}_{2}}{l_{2}} \frac{\partial}{\partial \xi_{2}}
$$

where $\nabla_{\xi}$ and $l_{i}$ are the surface gradient and scaling factors,

$$
l_{i}=\left\|\frac{\partial \mathbf{r}}{\partial \xi_{i}}\right\|=a_{i}\left(1-n k_{i}\right), \quad i=1,2 \quad \text { and } \quad l_{3}=\left\|\frac{\partial \mathbf{r}}{\partial n}\right\|=1
$$

The restriction of the surface gradient on the interface is defined as,

$$
\bar{\nabla}_{\xi}=\frac{\mathbf{e}_{1}}{a_{1}} \frac{\partial}{\partial \xi_{1}}+\frac{\mathbf{e}_{2}}{a_{2}} \frac{\partial}{\partial \xi_{2}}
$$

The Laplace operator then reads,

$$
\Delta=\frac{\partial^{2}}{\partial n^{2}}+\left(\nabla_{\xi} \cdot \mathbf{N}\right) \frac{\partial}{\partial n}+\Delta_{\xi}
$$

where

$$
\Delta_{\xi} \Phi=\nabla_{\xi} \cdot \nabla_{\xi} \Phi=\frac{1}{l_{1} l_{2}}\left(\frac{\partial}{\partial \xi_{1}}\left(\frac{l_{2}}{l_{1}} \frac{\partial}{\partial \xi_{1}} \Phi\right)+\frac{\partial}{\partial \xi_{2}}\left(\frac{l_{1}}{l_{2}} \frac{\partial}{\partial \xi_{2}} \Phi\right)\right)
$$

and

$$
\nabla_{\xi} \cdot \mathbf{N}=-\left(\frac{k_{1}}{1-n k_{1}}+\frac{k_{2}}{1-n k_{2}}\right)
$$

Restrictions of the above operators on the interface are defined as,

$$
\begin{gathered}
\bar{\Delta}=\frac{\partial^{2}}{\partial n^{2}}+\left(\bar{\nabla}_{\xi} \cdot \mathbf{N}\right) \frac{\partial}{\partial n}+\bar{\Delta}_{\xi} \\
\bar{\Delta}_{\xi} \Phi=\bar{\nabla}_{\xi} \cdot \bar{\nabla}_{\xi} \Phi=\frac{1}{a_{1} a_{2}}\left(\frac{\partial}{\partial \xi_{1}}\left(\frac{a_{2}}{a_{1}} \frac{\partial}{\partial \xi_{1}} \Phi\right)+\frac{\partial}{\partial \xi_{2}}\left(\frac{a_{1}}{a_{2}} \frac{\partial}{\partial \xi_{2}} \Phi\right)\right), \\
\bar{\nabla}_{\xi} \cdot \mathbf{N}=-\left(k_{1}+k_{2}\right)=-k
\end{gathered}
$$

where $k=k_{1}+k_{2}$ is the twice mean curvature of the interface.

Incompressibility condition

Consider an incompressible vector field,

$$
\mathbf{U}\left(n, \xi_{1}, \xi_{2}\right)=U_{n} \mathbf{N}+\mathbf{U}_{\perp}, \quad \nabla \cdot \mathbf{U}=0
$$

Then

$$
\nabla \cdot \mathbf{U}=\frac{\partial}{\partial n} U_{n}+\left(\nabla_{\xi} \cdot \mathbf{N}\right) U_{n}+\nabla_{\xi} \cdot \mathbf{U}_{\perp}
$$


In particular, the incompressibility condition implies,

$$
\left.\frac{\partial}{\partial n} U_{n}=-\left(\left(\nabla_{\xi} \cdot \mathbf{N}\right) U_{n}+\nabla_{\xi} \cdot \mathbf{U}_{\perp}\right)\right)
$$

Moreover, for $n=0$ we have,

$$
\frac{\partial}{\partial n} \bar{U}_{n}=k \bar{U}_{n}-\bar{\nabla}_{\xi} \cdot \overline{\mathbf{U}}_{\perp}
$$

where

$$
\overline{\mathbf{U}}\left(\xi_{1}, \xi_{2}\right)=\mathbf{U}\left(n=0, \xi_{1}, \xi_{2}\right),
$$

Time derivative

We define normal velocity of an interface $\left(V_{n}\right)$ and the restriction of the tangential velocity onto the interface $\left(\overline{\mathbf{V}}_{\perp}\right)$ as follows,

$$
V_{n}=\frac{\partial \mathbf{R}}{\partial t} \cdot \mathbf{N}, \quad \bar{V}_{\perp, i}=-\frac{\partial \mathbf{R}}{\partial t} \cdot \mathbf{e}_{i}
$$

In this setting the material time derivative of the scalar quantity $\phi\left(n, \xi_{1}, \xi_{2}, t\right)$ takes the following form,

$$
\frac{d \phi}{d t}=\mathcal{L} \phi-V_{n} \frac{\partial \phi}{\partial n}
$$

where

$$
\mathcal{L}_{t}=\partial_{t}+\mathbf{V}_{\perp} \cdot \nabla_{\xi}
$$

is the Lagrangian normal time derivative, that is

$$
\mathcal{L}_{t} \phi=\lim _{\Delta t \rightarrow 0} \frac{\phi\left(n, \xi_{1}+\Delta \xi_{1}, \xi_{2}+\Delta \xi_{2}, t+\Delta t\right)-\phi\left(n, \xi_{1}, \xi_{2}, t\right)}{\Delta t}
$$

Equation for the induced tangential velocity

It was shown in [14] that the restriction of the tangential velocity onto the interface $\overline{\mathbf{V}}_{\perp}$ obeys the following system of equations,

$$
\begin{gathered}
\frac{1}{a_{1} a_{2}}\left(\frac{\partial a_{2} \bar{V}_{\perp, 1}}{\partial \xi_{1}}+\frac{\partial a_{1} \bar{V}_{\perp, 2}}{\partial \xi_{2}}\right)=-k V_{n} \\
\frac{a_{1}}{a_{2}} \frac{\partial}{\partial \xi_{2}}\left(\frac{\bar{V}_{\perp, 1}}{a_{1}}\right)+\frac{a_{2}}{a_{1}} \frac{\partial}{\partial \xi_{1}}\left(\frac{\bar{V}_{\perp, 2}}{a_{2}}\right)=0
\end{gathered}
$$

Equations (A.21) and (A.22) in the coordinate free form read,

$$
\begin{gathered}
\bar{\nabla}_{\xi} \cdot \overline{\mathbf{V}}_{\perp}=-k V_{n} \\
\mathbf{e}_{1} \cdot\left(\nabla_{\xi} \overline{\mathbf{V}}_{\perp}\right) \cdot \mathbf{e}_{2}+\mathbf{e}_{2} \cdot\left(\nabla_{\xi} \overline{\mathbf{V}}_{\perp}\right) \cdot \mathbf{e}_{1}=0
\end{gathered}
$$

Therefore, the tangential (induced) flow $\mathbf{V}_{\perp}$ can be obtained from Eqs.(A.23),(A.24) when $V_{n}$ and $k$ are regarded as prescribed. In addition since the interface is moving in the normal direction $\overline{\mathbf{V}}_{\perp}$ must be zero at the lateral boundary of the interface. 


\section{References}

[1] M. Matalon. On Flame Stretch. Combust.Sci.Technol., 1983, Vol. 31, 169 -182.

[2] F.A. Williams. Combustion Theory. Benjamin/Cummings, Menlo Park, CA, 1985.

[3] B.J. Matkowsky, G.I. Sivashinsky. An Asymptotic Derivation of Two Models in Flame Theory Associated with the Constant Density Approximation. SIAM J. Appl. Math., 1979, Vol. 37, 686-699.

[4] M.L. Frankel, G.I. Sivashinsky. On the nonlinear thermal diffusive theory of curved flames. J. de Physique, 1987, Vol. 48, 25-28.

[5] M.L. Frankel, G.I. Sivashinsky. On the equation of a curved flame front. Physica D, 1988 , Vol. 30, 28- 42.

[6] G. Joulin, G.I. Sivashinsky, On the dynamics of nearly-extinguished non-adiabatic cellular flames. Combust. Sci. Technol., 1983, Vol. 31, 75-90.

[7] M.L. Frankel, P.V. Gordon, G.I. Sivashinsky. On disintegration of near-limit cellular flames. Phys. Letters A, 2003, Vol. 310, 389-392.

[8] M.L. Frankel, P. Gordon, G.I. Sivashinsky. A stretch-temperature model for flame-flow interaction. Phys. Letters A, 2007, Vol. 361, 356-359.

[9] G.I. Sivashinsky, C.K. Law, G. Joulin. On stability of premixed flames in stagnation -point flow. Combust. Sci. Technol., 1982, Vol. 28, 155-159.

[10] G.I.Sivashinsky. On self-turbulization of a laminar flame. Acta Astronaut., 1979, Vol. $6,569-591$.

[11] Y. Kim, M. Matalon. On the stability of near-equidiffusional strained premixed flames. Combust. Sci. Technol., 1990, Vol. 69, 85-97.

[12] Y. Kortsarts, I. Brailovsky, S. Gutman, G.I. Sivashinsky. On the stability of stretched flames. Combust. Theory Modelling, 1997, Vol. 1, 143 -156.

[13] G.K. Batchelor. An Introduction to Fluid Dynamics. Cambridge University Press, 1970.

[14] M. Matalon, C. Cui, J.K. Bechtold. Hydrodynamic theory of premixed flames: effects of stoichiometry variable transport coefficients and arbitrary reaction orders. J. Fluid Mech., 2003, Vol. 487,179-210.

[15] D.J. Struik. Lectures on Classical Differential Geometry. Dover, 1961. 